\title{
Reacting to Changes in Staging Designations in the 7th Edition of the AJCC Staging Manual
}

\author{
Daniel J. Boffa, MD' ${ }^{1}$ and Frederick L. Greene, $\mathrm{MD}^{2}$ \\ ${ }^{1}$ Department of Thoracic Surgery, Yale University School of Medicine, New Haven, CT; ${ }^{2}$ Department of General Surgery, \\ Carolinas Medical Center, Charlotte, NC
}

\section{THE “STAGE SHIFTER" PHENOMENON}

The American Joint Commission on Cancer (AJCC) staging manual was recently revised to reflect ongoing advances in the ability to stratify tumors by prognosis. ${ }^{1}$ For a number of tumor types, the $\mathrm{T}, \mathrm{N}$, and $\mathrm{M}$ staging parameters that form the backbone of the stage classification system were either redefined or reassigned to alternate stage groups. As a result, the same tumor might be given a stage classification in the revised (7th edition) manual that is either higher or lower than the stage classification of the previous (6th edition) staging manual. This apparent "upstaging" or "downstaging" of tumors by the revised manual has led to our referring to these tumors as the "stage shifters." For example, a 7.1-cm, node-negative non-small cell lung cancer (NSCLC) considered stage IB in the 6th edition is now considered stage IIB. A T3 esophageal cancer with a single celiac lymph node metastasis considered stage IV in the 6th edition has been reclassified stage IIIA in the 7th edition. In soft tissue sarcomas N1 tumors were reclassified from stage IV to stage III, and melanoma that is metastatic to lymph nodes from an unknown primary has been reclassified from stage IV to III.

The number of patients potentially affected by a stage reclassification, or the "stage shift" phenomenon is surprisingly large. Of the 54 nonlymphoid tumor types listed in the staging manual, 22 underwent stage classification changes that could result in stage shifters including lung, colorectal, esophageal, gastric, melanoma, and liver. With lung cancer alone, it is estimated that $10-17 \%$ of lung cancer patients in the United States would fall into 1 of the

(c) Society of Surgical Oncology 2010

Published Online: 10 November 2010

D. J. Boffa, MD

e-mail: daniel.boffa@yale.edu stage shift subgroups affecting more than 30,000 NSCLC patients each year. ${ }^{2}$

\section{POTENTIAL CONSEQUENCES OF STAGE RECLASSIFICATION}

Many clinicians consult stage-specific cancer treatment guidelines (NCCN, ASCO, etc.) as they formulate a treatment plan. The stage shift phenomenon may cause confusion as it is currently unclear whether patients should be treated according to the revised (7th edition) stage classification, or the previous version (6th edition). For example in lung cancer, node-negative tumors greater than $7 \mathrm{~cm}$ were previously considered stage IB, a stage where the benefits of chemotherapy after surgery are strongly suggested, but not proven. ${ }^{3}$ In the revised staging system, these tumors are classified as IIB, a stage for which adjuvant chemotherapy has been widely supported. ${ }^{4}$ In esophageal cancer, celiac lymph node metastases (a stage IV parameter in the 6th edition) were considered by many to represent a contraindication to surgery. However, celiac lymph node disease could be reclassified as stage II or III (stages in which surgery is widely considered the standard of care). On the other hand, well-differentiated, T3, node-negative squamous cell carcinomas of the esophagus (previously classified as stage IIA) have been treated with neoadjuvant chemotherapy followed by surgery. ${ }^{5}$ In the revised staging system, this tumor is reclassified to stage IB, a stage where many surgeons would proceed directly to surgery. It is unclear the extent to which clinicians will modify their treatment strategy in response to these stage classification changes.

We recently surveyed lung cancer clinicians for treatment recommendations for NSCLC stage shifter subgroups. ${ }^{6}$ Three NSCLC patient scenarios were first presented with the 6th edition stage designation. The same 3 patient scenarios were subsequently presented bearing the revised (7th edition) stage designation. Overall, $77 \%$ of the 
97 participating clinicians indicated that they would change treatment based solely on the change in stage designation, illustrating the potential for clinicians to respond to changes in stage classification by making changes in treatment strategy.

\section{MISINTERPRETING THE ASSOCIATION BETWEEN STAGE CLASSIFICATION AND TREATMENT EFFICACY}

Historically, clinical trials and other treatment studies have reported efficacy results according to tumor stage. This was both necessary and logical, as efficacy is not universal, and the staging parameters were the simplest mechanism to reproducibly characterize features (invasion, metastasis, etc.) felt likely to be related to efficacy. ${ }^{7}$ As a result, many treatment guidelines have adopted a stagespecific format to communicate treatment recommendations. Although generally useful, this practice encourages misinterpretation of the association between the stage classification system and treatment efficacy.

In other words, the stage classification nomenclature (I, II, III, IV) has simply been used by clinical trials to analyze data among tumors with similar attributes (size, invasiveness, nodal metastases, systemic metastases). While these tumor attributes themselves certainly may correspond to efficacy, it is illogical to consider treatment response to be embedded in the stage classification nomenclature. Therefore, reassigning tumor attributes to a new stage classification (as was done in the stage shift subgroups) is simply a change in nomenclature and does not imply any new insight on treatment efficacy. Therefore, changes in the stage classification should not be used as the sole motivation for changes in management strategy. For example, the clinical trials that demonstrated efficacy of preoperative chemotherapy for NSCLC with ipsilateral mediastinal lymph node metastases (stage classification IIIA) should not be used to now justify preoperative chemotherapy for 7.1$\mathrm{cm}$ tumors with hilar lymph node metastases (stage shift from IIB to IIIA), because the specific tumor attributes (large tumor with hilar lymph node metastases) were not adequately evaluated in these trials. ${ }^{8,9}$

\section{UNDERSTANDING THE DATA BEHIND THE CHANGES IN STAGE CLASSIFICATION SYSTEM}

Simply put, the changes in the stage classification system represent a more precise estimation of prognosis attributed to various tumor parameters $(\mathrm{T}, \mathrm{N}, \mathrm{M})$. Most revisions were informed by large staging databases designed and powered to evaluate survival data and tumor attributes. Importantly, the way in which tumors were treated was not a part of the analysis that motivated these changes. Therefore, even if a clinician sought to replicate the survival within the staging databases, without a careful evaluation of the treatment within the staging database, the clinician does not know what to start doing, or conversely what to stop doing.

The revised staging manual does provide updated survival data for the stage shift patients. For example, among the $17 \%$ of lung cancer patients estimated to fall into a stage shift subgroup, the average 5-year survival attributed to the revised stage classification (7th edition) differs from the previous stage classification by $10 \%$ (range $3-18 \%$ ). Realizing that prognosis is only a part of the complex treatment equation (including performance status, comorbidities, physician experience, and patient preferences), each clinician must gauge the influence updated prognostic information should have on each patient.

\section{RECOMMENDED REACTION TO CHANGES IN STAGE CLASSIFICATION (STAGE SHIFT)}

The optimal cancer treatment approach is multifactorial. Clinicians are advised whenever possible to formulate plans that are based on randomized clinical trials that study tumor attributes similar to the tumor and patient in question. We recognize that the change in prognostic information may be dramatic in some instances, and we certainly encourage clinicians to rethink the treatment their approach. However, the clinician should research the available literature and not simply apply treatment recommendations for tumors with a similarly named stage classification.

\section{REFERENCES}

1. AJCC, UICC. In: Edge SB, Byrd, DR, Compton CC, Fritz AG, Greene FL, Trotti A, editors. AJCC cancer staging manual. 7th ed. New York: Springer; 2009.

2. Goldstraw P, Crowley J, Chansky K, Giroux DJ, Groome PA, Rami-Porta R, et al. The IASLC Lung Cancer Staging Project: proposals for the revision of the TNM stage groupings in the forthcoming (seventh) edition of the TNM Classification of malignant tumours. $J$ Thorac Oncol. 2007;2:706-14.

3. Adjuvant chemotherapy, with or without postoperative radiotherapy, in operable non-small-cell lung cancer: two meta-analyses of individual patient data. Lancet. 2010;375:1267-77.

4. Arriagada R, Bergman B, Dunant A, Le Chevalier T, Pignon JP, Vansteenkiste J. Cisplatin-based adjuvant chemotherapy in patients with completely resected non-small-cell lung cancer. $N$ Engl J Med. 2004;350:351-60.

5. Gebski V, Burmeister B, Smithers BM, Foo K, Zalcberg J, Simes J. Survival benefits from neoadjuvant chemoradiotherapy or chemotherapy in oesophageal carcinoma: a meta-analysis. Lancet Oncol. 2007;8:226-34.

6. Boffa DJ, Detterbeck FC, Smith EJ, Rami-Porta R, Crowley J, Zelterman D, et al. Should the 7th edition of the lung cancer stage classification system change treatment algorithms in non-small cell lung cancer? J Thorac Oncol. 2010;5:1779-83. 
7. Detterbeck FC, Tanoue LT, Boffa DJ, Anatomy, biology and concepts, pertaining to lung cancer stage classification. J Thorac Oncol. 2009;4:437-43.

8. Rosell R, Maestre J, Font A, Moreno I, Molina F, Milla A, et al. A randomized trial of mitomycin/ifosfamide/cisplatin preoperative chemotherapy plus surgery versus surgery alone in stage IIIA nonsmall cell lung cancer. Semin Oncol. 1994;21:28-33.
9. Roth JA, Fossella F, Komaki R, Ryan MB, Putnam JB Jr, Lee JS, et al. A randomized trial comparing perioperative chemotherapy and surgery with surgery alone in resectable stage IIIA non-smallcell lung cancer. J Natl Cancer Inst. 1994;86:673-80. 